Check for updates

Cite this: RSC Adv., 2018, 8, 16362

\title{
Synthesis of novel (benzimidazolyl)isoquinolinols and evaluation as adenosine $A 1$ receptor tools $\uparrow$
}

Received 8th December 2017

Accepted 21st April 2018

DOI: $10.1039 / c 7 r a 13148 h$

rsc.li/rsc-advances
Sameek Singh, ${ }^{a}$ Samantha L. Cooper, ${ }^{\mathrm{b}}$ Jacqueline R. Glenn, ${ }^{\mathrm{b}}$ Jessica Beresford, ${ }^{\mathrm{b}}$ Lydia R. Percival, ${ }^{\mathrm{b}}$ Joel D. A. Tyndall, (D) ${ }^{\text {a }}$ Stephen J. Hill, ${ }^{\mathrm{b}}$ Laura E. Kilpatrick ${ }^{\mathrm{b}}$ and Andrea J. Vernall (D) *a

$G$ protein-coupled receptors (GPCRs) constitute the largest family of transmembrane receptors in eukaryotes. The adenosine $A_{1}$ receptor $\left(A_{1} A R\right)$ is a class $A$ GPCR that is of interest as a therapeutic target particularly in the treatment of cardiovascular disease and neuropathic pain. Increased knowledge of the role $A_{1} A R$ plays in mediating these pathophysiological processes will help realise the therapeutic potential of this receptor. There is a lack of enabling tools such as selective fluorescent probes to study $A_{1} A R$, therefore we designed a series of (benzimidazolyl)isoquinolinols conjugated to a fluorescent dye (31-35, 42-43). An improved procedure for the synthesis of isoquinolinols from tetrahydroisoquinolinols via oxidation with 2,3-dichloro-5,6-dicyano-1,4-benzoquinone (DDQ) and atmospheric oxygen is reported. This synthetic method offers advantages over previous metal-based methods for the preparation of isoquinolinols and isoquinolines, which are important scaffolds found in many biologically active compounds and natural products. We report the first synthesis of the (benzimidazolyl)isoquinolinol compound class, however the fluorescent conjugates were not successful as $A_{1} A R$ fluorescent ligands.

\section{Introduction}

Adenosine receptors (ARs) are class A G protein-coupled receptors (GPCRs), classified in four distinct subtypes $-A_{1}$, $A_{2 A}, A_{2 B}$ and $A_{3}$. Adenosine $A_{1}$ receptor $\left(A_{1} A R\right)$ is expressed throughout the human body with predominant distribution in brain, heart, kidney and adipose tissue and is an attractive therapeutic target. ${ }^{1}$ To this end, $\mathrm{A}_{1} \mathrm{AR}$ ligands are of interest and are being developed to treat various pathological conditions such as atrial fibrillation, angina pectoris, congestive heart failure, diabetes, neuropathic pain and renal disorders. ${ }^{2}$ Fluorescent ligands have emerged as useful and powerful tools to study real-time and live cell dynamic processes of GPCRs. ${ }^{3}$ Fluorescent ligands have been developed for ARs, ${ }^{4}$ including some with high AR subtype selectivity for $\mathrm{A}_{3} \mathrm{AR} .{ }^{5}$ There is one report of fluorescent dansyl-linked $\mathrm{N}^{6}$ NECA derivatives with subtype selectivity for rat $\mathrm{A}_{1} \mathrm{AR},{ }^{6}$ however the short excitation wavelength of the dansyl fluorophore is not ideal for many applications. When the same research group conjugated the lead pharmacophore-linker to longer excitation wavelength fluorophores $\mathrm{A}_{3} \mathrm{AR}$ selective probes ensued. ${ }^{6}$ Therefore there

${ }^{a}$ School of Pharmacy, University of Otago, PO Box 56, Dunedin 9054, New Zealand. E-mail: andrea.vernall@otago.ac.nz; Tel: +6434794518

${ }^{b}$ Physiology, Pharmacology and Neuroscience Division, School of Life Sciences, University of Nottingham, Nottingham, NG7 2UH, UK

$\dagger$ Electronic supplementary information (ESI) available. See |DOI: $10.1039 / \mathrm{c} 7 \mathrm{ra} 13148 \mathrm{~h}$ remains a need to develop fluorescent ligands with high subtype selectivity for $\mathrm{A}_{1} \mathrm{AR}$.

One approach to the design of a fluorescent ligand is to identify an existing pharmacophore with the requisite selectivity and affinity for the target receptor, and then identify a location that a linker and fluorophore can be attached. ${ }^{5}$ With this design in mind, it is then necessary to synthesise and test a range of pharmacophore, pharmacophore-linker and fluorescent ligands as modifications have the potential to alter the affinity and efficacy of the parent pharmacophore. In an effort to develop a selective $A_{1} A R$ fluorescent ligand we chose to avoid a xanthine amine congener (XAC)-based pharmacophore because of subtype selectivity concerns as a range of XACfluorescent conjugates have been reported as either non AR subtype selective or $\mathrm{A}_{3} \mathrm{AR}$ selective. ${ }^{7}$ Cosimelli et al. recently reported a series of (benzimidazolyl)isoquinolines as selective

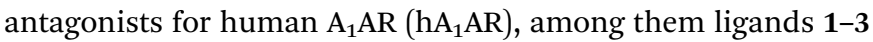
(Fig. 1), ${ }^{8}$ which were used as the inspiration for the fluorescent ligand design reported herein.

Structure-activity relationships (SAR) from the Cosimelli et al. study indicated small, lipophilic benzimidazole substituents such as methyl and ethylthio groups in some positions improved $\mathrm{A}_{1} \mathrm{AR}$ affinity and in others abolished it. ${ }^{8}$ This sensitivity to slight positional change, along with potential buried, lipophilic interactions these groups could be making with the $\mathrm{A}_{1} \mathrm{AR}$ to enhance affinity led us to discount linker substitution from the benzimidazole ring. Since 1,3-substituted isoquinoline analogues such as VUF5455 (ref. 9) and LUF6096 (ref. 10) have 


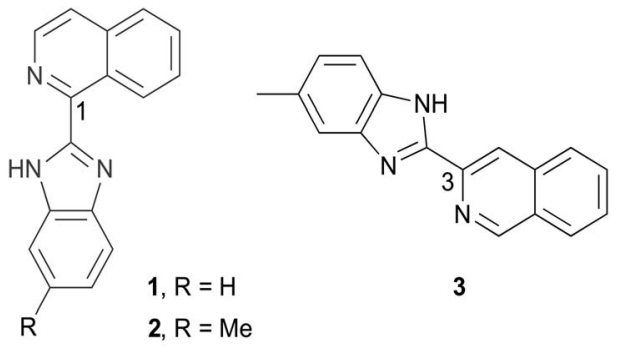

\begin{tabular}{cccc} 
& $\mathrm{hA}_{1} \mathrm{AR}$ & $\mathrm{hA}_{2 \mathrm{~A}} \mathrm{R}$ & $\mathrm{hA}_{3} \mathrm{AR}$ \\
& $K_{\mathrm{i}}(\underline{\mathrm{nM}})$ & $K_{\mathrm{i}}(\underline{\mathrm{nM}})$ & $K_{\mathrm{i}}(\mathrm{nM})$ \\
\hline $\mathbf{1}$ & $14.1 \pm 1.6$ & $>10,000$ & $>1,000$ \\
$\mathbf{2}$ & $6.6 \pm 0.5$ & $>10,000$ & $>1,000$ \\
$\mathbf{3}$ & $3.5 \pm 0.3$ & $>10,000$ & $264.7 \pm 23$
\end{tabular}

Fig. $1 A_{1} A R$ subtype selective (benzimidazolyl)isoquinolines with previously reported AR affinities. ${ }^{8}$

been reported as allosteric ligands for $\mathrm{A}_{3} \mathrm{AR}$, albeit with quite different substituents, we chose to instead explore the previously unexplored isoquinoline C5-8 positions for linker attachment. $\mathrm{A}_{1} \mathrm{AR}$ ligands such as 1-3 lack a reactive functional group for linker and subsequently fluorophore attachment in these positions, therefore we designed a series of (benzimidazolyl)isoquinolinols. To the best of our knowledge, there are no previous literature reports on the synthesis of (benzimidazolyl)isoquinolinols. The hydroxyl group offers accessible chemistry for linker attachment, e.g. via an ether bond that remains unionised at physiological pH. Succinimidyl-6-[2- $(p-\{(E)-2-[4,4-d i f l u o r o-5-$ (2-thienyl)-3a,4a-diaza-4-bora-s-indacen-3-yl]-ethenyl $\}$ phenoxy) acetylamino]hexanoate (BODIPY630/650-SE) was chosen as the fluorophore for coupling because of its success in developing fluorophores for studying GPCRs in previous studies ${ }^{\mathbf{4 , 5 , 1 1}}$ and its suitable spectroscopic properties as an acceptor in bioluminescence resonance energy transfer (BRET) assays. ${ }^{12}$

We envisaged using the Pictet-Spengler reaction ${ }^{13}$ in our synthesis to form a hydroxytetrahydroisoquinoline carboxylic acid followed by aromatisation to give a hydroxyisoquinoline carboxylic acid. This required an aromatisation reaction tolerant of functional groups such as a phenol, amine and carboxylic acid. Although isoquinoline is an important pharmacophore in various natural products, metal co-ordination ligands and marketed drugs such as papaverine, ripasudil, quinisocaine and moxaverine, a general method for the construction of isoquinolines with broad functional group tolerance, including the synthesis of isoquinolinols, is lacking. Aromatisation of tetrahydroisoquinoline to isoquinoline is usually carried out using heavy metals such as palladium with harsh conditions with poor functional group tolerance. ${ }^{\mathbf{1 4 - 2 1}}$ Protection/deprotection of functional groups is another common strategy employed for successful aromatisation to prepare functionalised isoquinolines. ${ }^{22,23}$ Use of 2,3-dichloro5,6-dicyano-1,4-benzoquinone (DDQ) has been reported for the aromatisation of tetrahydroisoquinoline $e^{22,24,25}$ and dihydroisoquinoline $\mathrm{e}^{26,27}$ to isoquinolines at high temperature. In this study, we explored the use of DDQ to aromatise tetrahydroisoquinols to isoquinolinols under mild conditions as an alternate to heavy metal-based or harsh reaction conditions.

\section{Results and discussion}

The synthesis of 1-substituted 6-hydroxyl tetrahydroisoquinoline 5 began with attempts to carry out a Pictet-Spengler reaction of 3-(2-aminoethyl)phenol hydrobromide 4 with glyoxylic acid monohydrate following the procedure Li et al. ${ }^{28}$ (no spectroscopic data provided for 5, ref. 29), however these were unsuccessful. Pleasingly, following the synthesis of $\mathbf{5}$ reported by Maillard et al. ${ }^{29}$ tetrahydroisoquinoline $\mathbf{5}$ was obtained in good yield, the point of difference between the Li et al. ${ }^{28}$ and Maillard et al. ${ }^{29}$ procedures being addition of triethylamine to the amine hydrobromide salt prior to addition of glyoxylic acid monohydrate (Scheme 1). The neutralisation presumably meant the imine could form more readily and hence the Pictet-Spengler reaction could proceed. Attempts to aromatise carboxylic acid 5 using DDQ and isolate the hydroxyisoquinoline carboxylic acid (using optimised conditions described below) were problematic, in part due to difficulty analysing and purifying the polar carboxylic acid. Therefore $\mathbf{5}$ was esterified to give methyl ester 6 (compound 6 was previously reported by Ma et $a .^{30}$ but with no spectroscopic data). Synthesis of the 3substituted tetrahydroisoquinolinol series began with PictetSpengler condensation of (+/-)-m-tyrosine 8 with formaldehyde to give 9 according to a literature procedure reporting the synthesis of $9,^{31}$ followed by esterification to give 10. The synthesis of methyl ester $\mathbf{1 0}$ from $\mathbf{9}$ has previously been reported $^{18,25,32-34}$ however none of these reports include spectroscopic data for 10. The first attempt to synthesise the 7-hydroxyl tetrahydroisoquinoline series via condensation of tyramine with glyoxylic acid monohydrate according to the procedure for 5 (except without trimethylamine as the amine was not a salt) was not successful. Failure of this reaction was attributed to the lack of an activating group in tyramine, compared to the presence of an activating para hydroxyl relative to the point of condensation for 4 and 8. Instead, Fmoc-protected tyramine 12 was reacted with glyoxylic acid monohydrate to give a carboxylic acid according to the synthesis of this carboxylic acid reported by Maillard et al. ${ }^{29}$ which was esterified to give $\mathbf{1 3}$ in low yield over 2 steps. NMR spectra of $\mathbf{1 3}$ showed a mixture of isomers that were confirmed as rotamers rather than regioisomers via variable temperature NMR experiments.

There is one report of the aromatisation of an unprotected hydroxytetrahydroisoquinoline (10) with DDQ in patent literature (conversion of $\mathbf{1 0}$ to 11, no spectroscopic data for $\mathbf{1 1}$ provided $^{25}$ ) however in our hands all initial attempts to aromatise $\mathbf{6}$ or $\mathbf{1 0}$ using DDQ with varying temperatures and solvents and with either oxygen excluded or in a closed reaction vessel failed. When 6 and DDQ were dissolved in THF and dioxane and heated to $100{ }^{\circ} \mathrm{C}$ under a $\mathrm{N}_{2}$ atmosphere the partially oxidised methyl 6-hydroxy-3,4dihydroisoquinoline-1-carboxylate was isolated as the major product. However, at moderate temperature and with vigorous stirring in a flask open to the atmosphere, 6 and DDQ were reacted to give the desired product 7 in moderate yield. To our knowledge, 


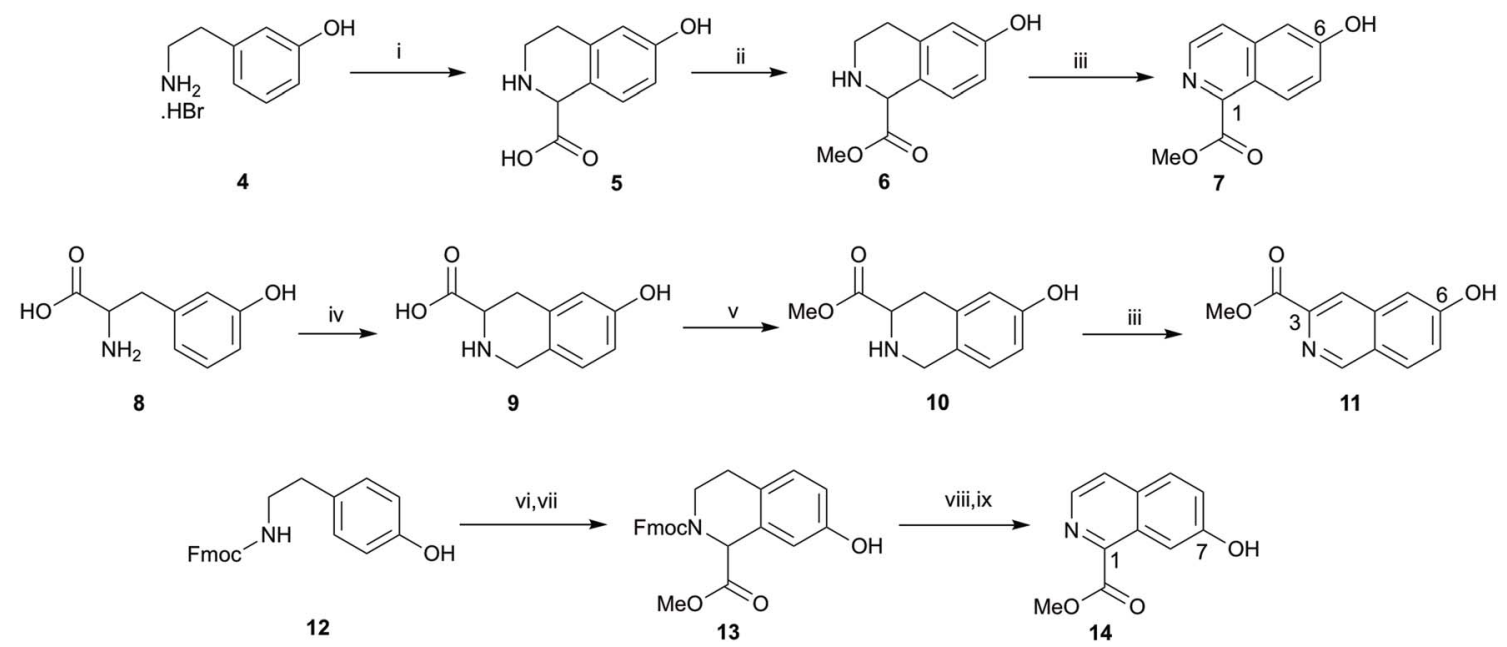

Scheme 1 Synthesis of isoquinolines. Reagents and conditions: (i) $\mathrm{Et}_{3} \mathrm{~N}$, glyoxylic acid monohydrate, $\mathrm{EtOH}, 71 \%$; (ii) $\mathrm{SOCl}, \mathrm{MeOH}_{2} 65{ }^{\circ} \mathrm{C}$, quantitative; (iii) $\mathrm{DDQ}$, THF, 1,4 dioxane, $45^{\circ} \mathrm{C}$, 49-52\%; (iv) $\mathrm{HCl}, \mathrm{H}_{2} \mathrm{O}$, formaldehyde (37 wt\% in $\mathrm{H}_{2} \mathrm{O}$ ) $100{ }^{\circ} \mathrm{C}, 48 \%$; (v) $\mathrm{H}_{2} \mathrm{SO}_{4}, \mathrm{MeOH}^{2} 96 \%$; (vi) glyoxylic acid monohydrate, $\mathrm{H}_{2} \mathrm{SO}_{4}, \mathrm{CH}_{3} \mathrm{CO}_{2} \mathrm{H}$; (vii) $\mathrm{SOCl}_{2}, \mathrm{MeOH}, 18 \%$ over two steps; (viii) DMSO, MeOH, $60{ }^{\circ} \mathrm{C}$; (ix) DDQ, THF, 1,4 dioxane, $45^{\circ} \mathrm{C}, 61 \%$ over two steps.

isoquinoline 7 has only been reported once before in the literature in a Japanese patent ${ }^{35}$ but no spectroscopic data was provided. The procedure to convert 6 to 7 was also used to successfully aromatise 10 to 11. Since the aromatised product did not form without vigorous stirring open to the atmosphere it is likely that DDQ mediates formation of a dihydroisoquinoline, which is then aromatised by oxygen as the active oxidant. Oxygen has previously been reported as an oxidant in aromatisation reactions ${ }^{36,37}$ and indeed Dong et al. $^{38}$ used a base in dimethyl sulfoxide (DMSO) along with oxygen from air for conversion of $\mathrm{N}$-tosyltetrahydroisoquinolines into isoquinolines. Dong et al. ${ }^{38}$ also isolated an imine under an argon atmosphere instead of the isoquinoline product, analogous to the partially oxidised imine intermediate (methyl 6-hydroxy-3,4-dihydroisoquinoline-1-carboxylate) isolated in our study with exclusion of air. Formation of an iminium ion intermediate (3,4-dihydroisoquinoline) has also been reported for DDQ-based cross-dehydrogenative coupling reactions of tetrahydroisoquinolines. ${ }^{39}$

Diethylamine was initially used for Fmoc deprotection of $\mathbf{1 3}$ however subsequent aromatisation with DDQ provided poor yield of 14, most likely due to residual diethylamine. High temperature NMR spectra in DMSO- $d_{6}$, carried out to study rotamers of 13 revealed cleavage of Fmoc, and indeed there is precedent in the literature that Fmoc cleavage can be conducted in neat DMSO at high temperature. ${ }^{40}$ Thus, Fmoc cleavage of $\mathbf{1 3}$ in DMSO, followed by DDQ and air mediated aromatisation afforded 14 in comparable yield to 7 and 11. The moderate yield of DDQ-air mediated aromatisation is unclear but may be due to low solubility in organic solvent of these compounds and susceptibility to methyl ester hydrolysis during basic work up.

Hydrolysis of 7 and $\mathbf{1 4}$ under basic conditions provided $\mathbf{1 5}$ and 16, which were condensed with $o$-phenylenediamine or 2,4diaminotoluene in hot polyphosphoric acid (PPA) to afford 1(benzimidazolyl)isoquinolinols 17-19 in low yield (Scheme 2). This low yield was likely due to a number of factors, including poor double dehydration yield and a difficult isolation of a product with low organic solvent solubility from the viscous reaction mixture. NMR spectra of 17-19 showed a mixture of conformers, which was elucidated to be tautomers using variable solvent and high temperature NMR experiments and high performance liquid chromatography (HPLC) (discussed in ESI $\dagger$ ). The phenolic group of 17-19 was reacted with tert-butyl bromoacetate to afford corresponding tert-butyl esters 20-22, which were reacted with trifluoroacetic acid to give carboxylic acids 23-25. Carboxylic acid 24 was coupled to three different amino linkers - $\mathrm{a} \mathrm{C}_{8}$ alkyl, short polyethylene glycol (PEG) or LAla-L-Ala short peptide to give 27,28 or 29 respectively. Carboxylic acid 23 underwent amide coupling to give PEGlinked 26 while the 7-substituted carboxylic acid 25 was converted to PEG-linked 30. These linkers were selected to explore variations in the physicochemical properties of the resulting fluorescent probes, in particular in light of lipophilicity and non-specific membrane binding. Boc deprotection of 26-30 provided amines that were reacted with BODIPY630/650-SE to give fluorescent conjugates 31-35. 3-(Benzimidazolyl) isoquinolinol-based fluorescent conjugates 42 and 43 were synthesised using analogous methodology (Scheme 3). Absorption and emission spectra of fluorescent compounds 3135 and 42-43 showed excitation maxima at $624 \mathrm{~nm}$ and emission maxima at either $641 \mathrm{~nm}$ or $642 \mathrm{~nm}$ (detailed in ESI $\dagger$ ).

\section{Pharmacology}

Fluorescent ligands 31-35 and 42-43 were analysed for their ability to bind to $\mathrm{A}_{1} \mathrm{AR}$ according to a previously reported bioluminescence resonance energy transfer (NanoBRET) saturation binding assay using the novel luciferase NanoLuc (NLuc; Promega Corporation, USA). ${ }^{\mathbf{1 2}}$ HEK293 cell lines stably transfected with $\mathrm{N}$-terminal NLuc labelled $\mathrm{A}_{1} \mathrm{AR}$ were treated with increasing concentrations of the (benzimidazolyl)isoquinolinol fluorescent ligands, in the presence and absence of $1 \mu \mathrm{M}$ DPCPX (a high affinity, non-fluorescent, selective $\mathrm{A}_{1} \mathrm{AR}$ antagonist) to determine levels of non-specific binding. 


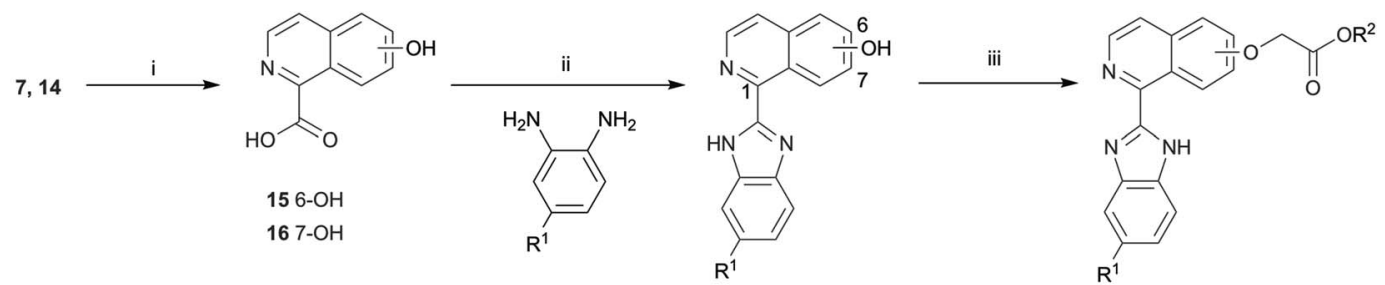

$176-\mathrm{OH}, \mathrm{R}^{1}=\mathrm{H}$

$186-\mathrm{OH}, \mathrm{R}^{1}=\mathrm{CH}_{3}$

$197-\mathrm{OH}, \mathrm{R}^{1}=\mathrm{CH}_{3}$

20 6-O-linker, $R^{1}=H, R^{2}=t B u$

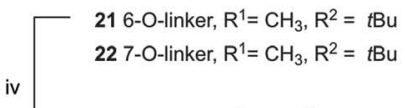

23 6-O-linker, $\mathrm{R}^{1}=\mathrm{H}, \mathrm{R}^{2}=\mathrm{H}$

24 - - -linker, $\mathrm{R}^{1}=\mathrm{CH}_{3}, \mathrm{R}^{2}=\mathrm{H}$

25 7-O-linker, $\mathrm{R}^{1}=\mathrm{CH}_{3}, \mathrm{R}^{2}=\mathrm{H}$
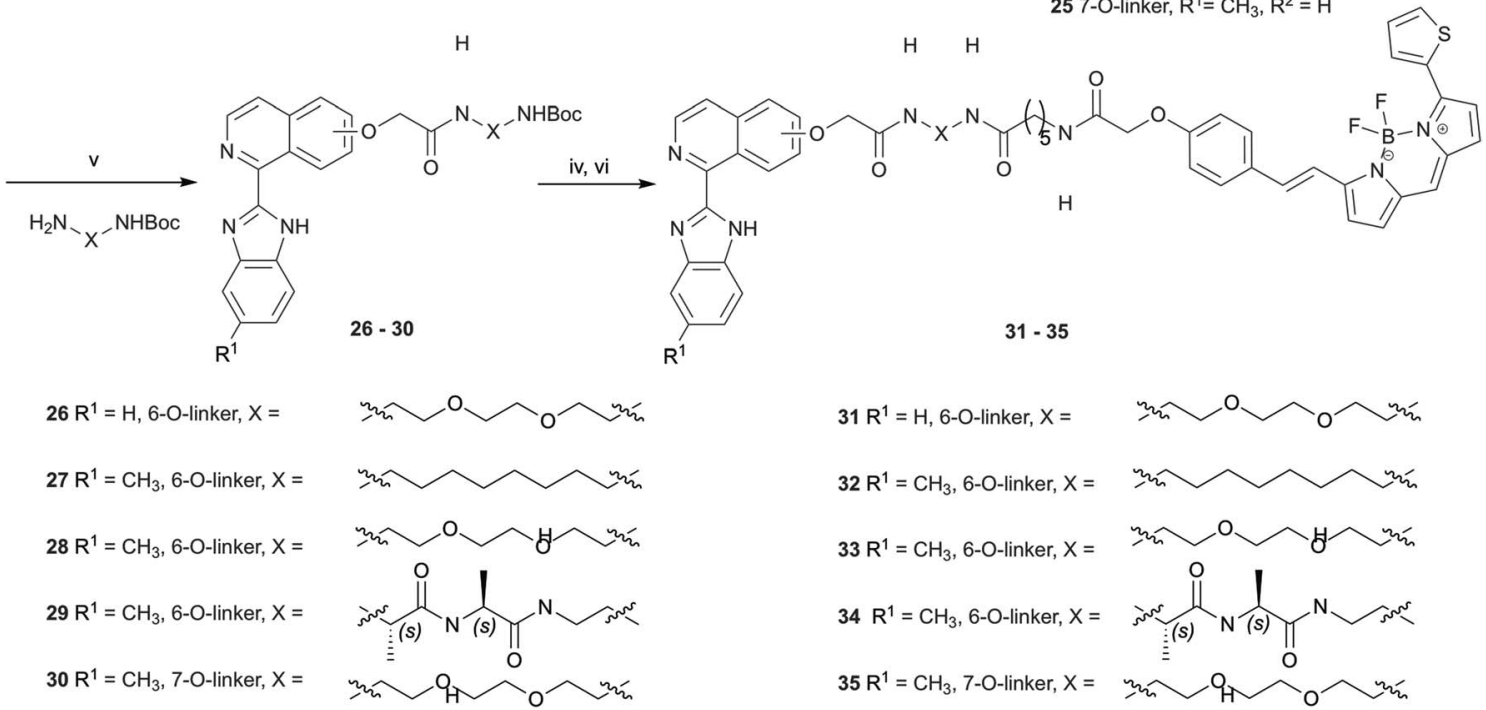$$
32 \mathrm{R}^{1}=\mathrm{CH}_{3}, 6-\mathrm{O} \text {-linker, } \mathrm{X}=\text { 经 }
$$$$
33 \mathrm{R}^{1}=\mathrm{CH}_{3}, 6-\mathrm{O} \text {-linker, } \mathrm{X}=\text { 等 }
$$$$
34 \mathrm{R}^{1}=\mathrm{CH}_{3}, 6 \text {-O-linker, } \mathrm{X}=\text { = }
$$$$
35 \mathrm{R}^{1}=\mathrm{CH}_{3}, 7-\mathrm{O} \text {-linker, } \mathrm{X}=\text { 经 }
$$

Scheme 2 Synthesis of 1-(1H-benzimidazol-2-yl)isoquinolinols. Reagents and conditions: (i) LiOH, THF, $\mathrm{H}_{2} \mathrm{O}, 70-96 \%$; (ii) PPA, 250 ${ }^{\circ} \mathrm{C}, 21-38 \%$; (iii) tert-butyl bromoacetate, $\mathrm{K}_{2} \mathrm{CO}_{3}, \mathrm{THF}, 60{ }^{\circ} \mathrm{C}, 66-96 \%$; (iv) TFA, DCM, 60\% - quantitative; (v) HATU, DIPEA, DMF, 29-69\%; (vi) BODIPY630/ 650-SE, DIPEA, DMF, 33-84\%.

Unfortunately, all ligands 31-35 and 42-43 did not show any indication of binding to $\mathrm{A}_{1} \mathrm{AR}$, for example data shown for 31 and 33 (Fig. 2a and b) (other compound data in ESI Fig. S16†).
Fluorescent ligands 31-34 were then analysed for their ability to bind to NLuc-labelled $\mathrm{A}_{3} \mathrm{AR}$ in analogous NanoBRET experiments, using $\mathrm{A}_{3} \mathrm{AR}$ selective MRS1220 to establish non-specific

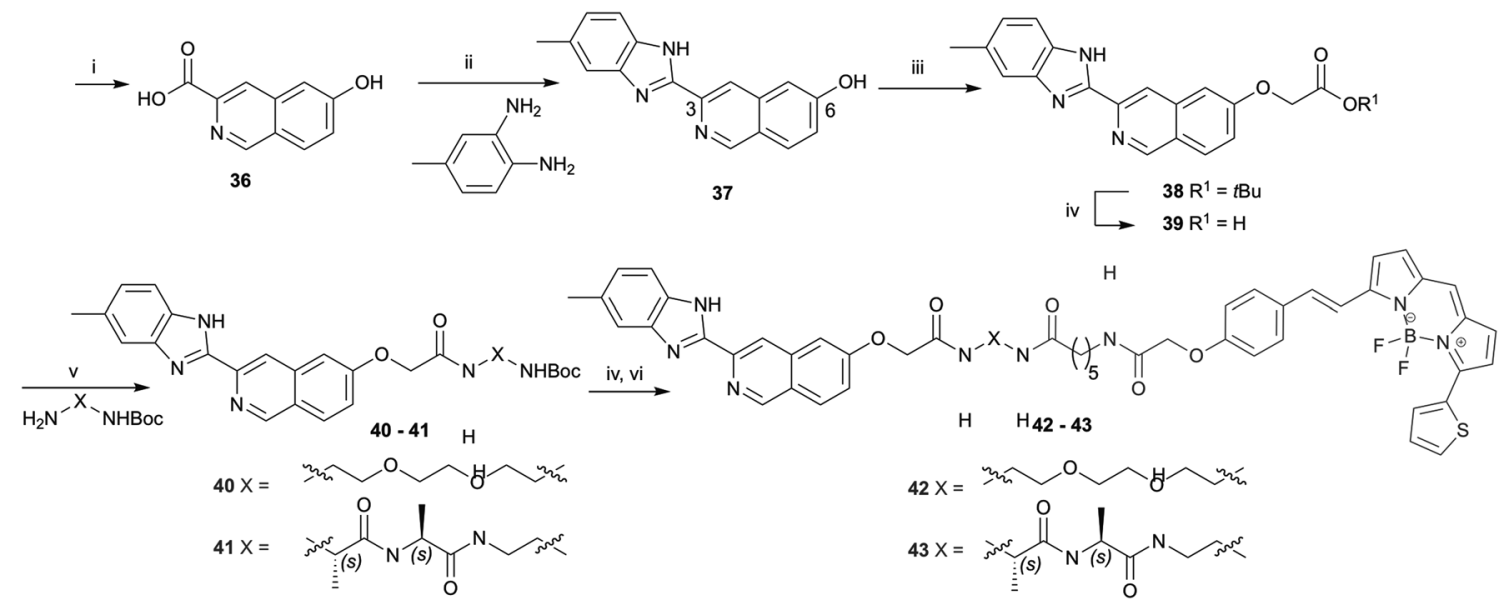

Scheme 3 Synthesis of 3-(1H-benzimidazol-2-yl)isoquinolinols. Reagents and conditions: (i) LiOH, THF, $\mathrm{H}_{2} \mathrm{O}, 90 \%$; (ii) PPA, $250{ }^{\circ} \mathrm{C}, 26 \%$; (iii) tertbutyl bromoacetate, $\mathrm{K}_{2} \mathrm{CO}_{3}, \mathrm{THF}, 60{ }^{\circ} \mathrm{C}, 94 \%$; (iv) TFA, DCM, quantitative; (v) HATU, DIPEA, DMF, 37-39\%; (vi) BODIPY630/650-SE, DIPEA, DMF, $73-86 \%$ 


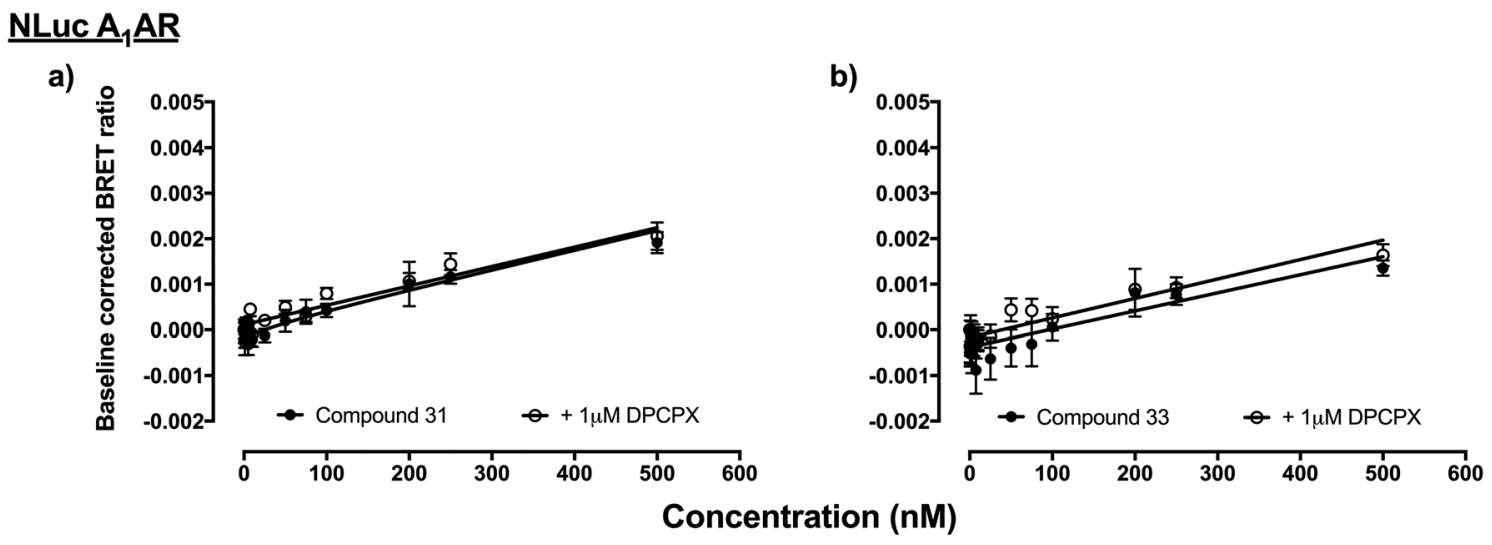

\section{NLuc $A_{3} \underline{A R}$}

c)

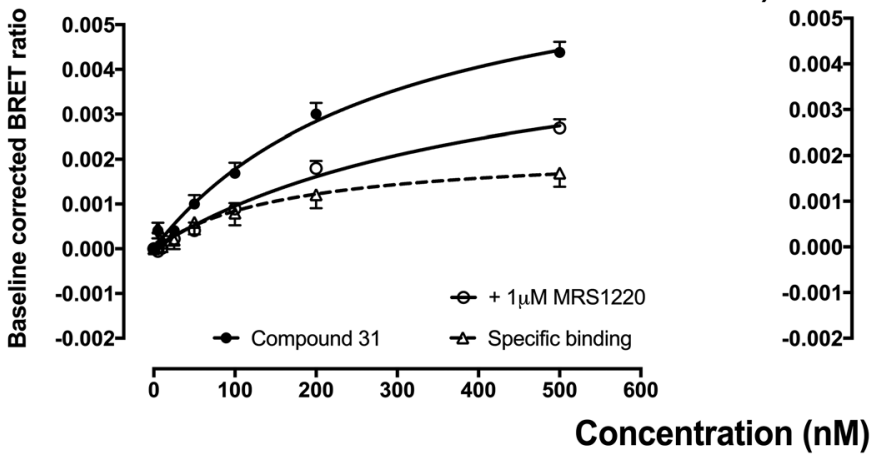

d)

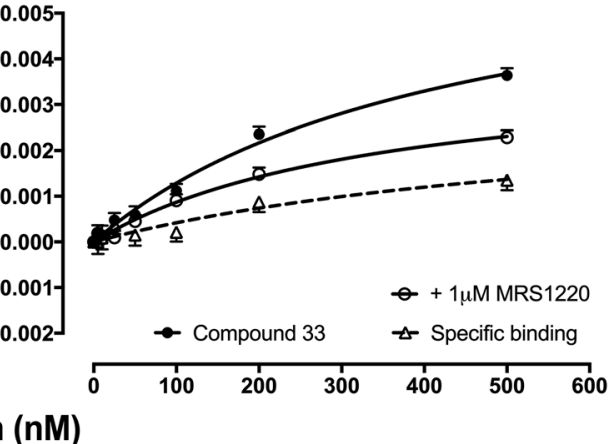

Fig. 2 HEK293 cells stably transfected with N-terminally NLuc-tagged $A_{1} A R$ or $A_{3} A R$ were treated with increasing concentrations of fluorescent ligand and the BRET ratio measured after direct addition of the NLuc substrate furimazine $(10 \mu \mathrm{M}) .{ }^{12}$ Non-specific binding was assessed in the absence and presence of $1 \mu \mathrm{M}$ DPCPX (NLuc- $A_{1} A R$ ) or $1 \mu \mathrm{M}$ MRS1220 (NLuc-A AR). Pooled data of raw BRET ratio's were baseline corrected (minus vehicle + furimazine BRET ratios) so that data are expressed as fold increase in BRET ratios over basal. (a) NLuc- $A_{1} A R$ and 31 , (b) NLuc$A_{1} A R$ and 33, (c) NLuc- $A_{3} A R$ and 31 and (d) NLuc- $A_{3} A R$ and 33. Data represents four-seven independent experiments (in triplicate).

binding. Ligands 32 and $\mathbf{3 4}$ did not show any specific binding to NLuc- $\mathrm{A}_{3} \mathrm{AR}$ (data not shown), while 31 and to a lesser extent 33 (Fig. 2c and d) showed a small degree of binding to NLuc- $\mathrm{A}_{3} \mathrm{AR}$. However, the specific binding window was small and the standard error's of the calculated $K_{\mathrm{d}}$ values for specific binding to NLuc-A 3 AR were large $\left(31 K_{\mathrm{d}}=162 \pm 65.5 \mathrm{nM}, n=5 ; 33 K_{\mathrm{d}}=\right.$ $534 \pm 254 \mathrm{nM}, n=4)$.

This NanoBRET assay is an excellent way to assess new fluorescent ligands $\mathrm{s}^{\mathbf{4 1}}$ since it measures the interaction of the fluorescent ligand and receptor directly without relying on the ability of the test ligand to compete with another tracer such as a radioligand. In this study the level of fluorescent ligand nonspecific/membrane-binding was defined by measuring BRET in the presence, for $\mathrm{A}_{3} \mathrm{AR}$, of a competing high concentration of unlabelled MRS 1220. Therefore this measure of specificity is defined by the ability of MRS $1220(a[1,2,4]$ triazolo[1,5- $c]$ quinazolinyl pharmacophore) to prevent binding of the fluorescent compounds, which implies each would be interacting with the same or an overlapping region of the ligand binding pocket of the receptor. It is clear from Fig. $2 c$ and $d$, however, that the non-specific binding component (obtained in the presence of MRS 1220) is not linear and is better fit with a saturable binding curve. It therefore remains a possibility (along with non-specific membrane interactions) that $\mathbf{3 1}$ and $\mathbf{3 3}$ may be interacting with an additional region of $\mathrm{A}_{3} \mathrm{AR}$ distinct from the orthosteric binding site of MRS 1220.

A possible explanation for the lack of binding of fluorescent ligands 31-35 and 42-43 to NLuc $A_{1} A R$, is steric occlusion of binding due to the presence of the N-terminal NanoLuc tag. To investigate this, NanoBRET was used to quantify the specific binding of CA200645, a known fluorescent non selective AR ligand at NLuc $\mathrm{A}_{1} \mathrm{AR}$. Saturable binding was observed, with minimal non-specific binding and a large observation window (Fig. 3A; $K_{\mathrm{d}}=110 \mathrm{nM}+18.46 n=5$ ) consistent with previous NanoBRET observations of CA200645 binding to NLuc $A_{1}$ AR or NLuc $A_{3} A R .^{12}$ NanoBRET assay plates were then washed and imaged using a IX Ultra confocal plate reader (MetaXpress, Molecular Devices) with fluorescence intensity quantified per well using a modified multiple wavelength cell scoring algorithm. ${ }^{42}$ Saturable binding of CA200645 was observed at NLuc $\mathrm{A}_{1} \mathrm{AR}\left(K_{\mathrm{d}}=189.3 \mathrm{nM} \pm 69.6 ; n=4\right.$, data not shown), with a $K_{\mathrm{d}}$ value consistent with that measured by NanoBRET (Fig. 3A). The binding of CA200645 to untagged $\mathrm{A}_{1}$ AR stably expressed in HEK293, was also quantified using this fluorescent imagingbased technique with the resultant estimated binding affinity comparable to that seen for NLuc tagged $\mathrm{A}_{1} \mathrm{AR}(144.8 \mathrm{nM} \pm 63.6$; $n=3$; data not shown). From these results it can be concluded that the NanoLuc tag does not occlude binding of CA200645 to 
A

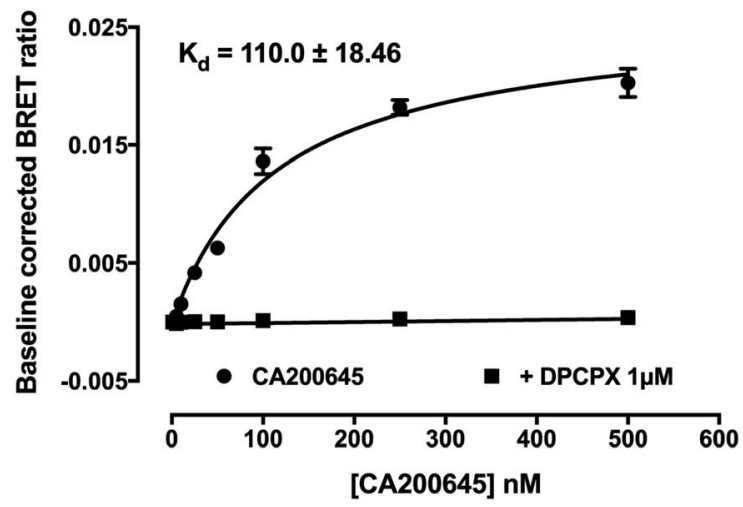

B

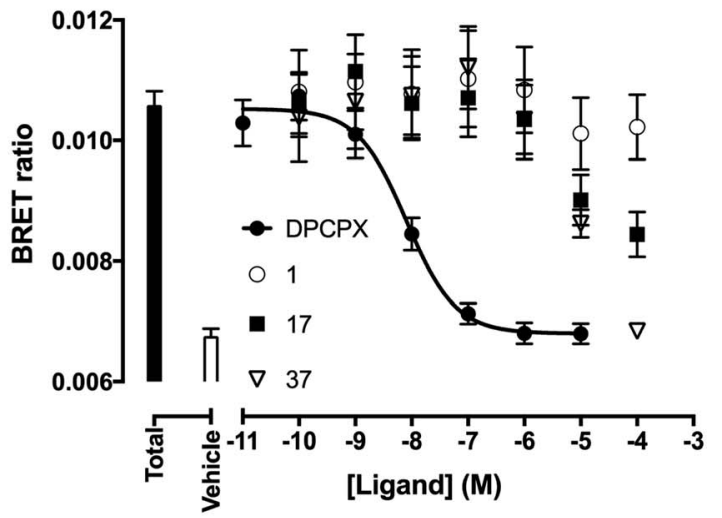

Fig. 3 HEK293 cells stably expressing N-terminal NLuc tagged $A_{1} A R$ were treated with increasing concentrations of CA200645 (5-500 $\mathrm{nM}$; $1 \mathrm{~h}$ at $37^{\circ} \mathrm{C}$; A). Non specific binding was defined using $1 \mu \mathrm{M}$ DPCPX, an $\mathrm{A}_{1} \mathrm{AR}$ selective antagonist. The NanoLuc substrate furimazine was added (10 $\mu \mathrm{M})$, with luminescence and fluorescence emissions recorded using a Pherastar FS. Data was pooled from independent experiments $(n=5)$ and baseline corrected (minus vehicle + furimazine BRET ratios) so that data are expressed as fold increase in BRET ratios over basal and where appropriate fit using one site saturation binding (mean \pm SEM). For competition experiments (B), NLuc $A_{1} A R$ were co-incubated with a fixed concentration of CA200645 and increasing concentrations of unlabelled ligand ( $1 \mathrm{~h}$ at $\left.37^{\circ} \mathrm{C}\right)$. Total CA200645 binding and vehicle are shown by the black and white bars respectively. Data were pooled from five independent experiments and are expressed as mean \pm SEM.

the receptor, therefore it seems unlikely this tag is the reason for the poor binding of fluorescent ligands 31-35 and 42-43.

In light of the poor binding of fluorescent ligands 31-35 and 42-43 to $A_{1} A R$ as measured using NanoBRET competition binding experiments, a subset of (benzimidazolyl)isoquinolinols described in Schemes 2 and 3 (1, 17-19, 28, 30, 37 and 40) and literature reference compound 1 were tested using NanoBRET competition assays. HEK293 NLuc $\mathrm{A}_{1} \mathrm{AR}$ cells were treated with a fixed concentration of CA200645 (25 nM) and increasing concentrations of unlabelled ligand. The $\mathrm{A}_{1} \mathrm{AR}$ selective antagonist DPCPX was used as a positive control. No displacement of CA200645 binding was observed with benzimidazolyl)isoquinolinols 1, 17-19, 28, 30, 37 and 40 until ligand concentrations were in excess of $10^{-6} \mathrm{M}$ (Fig. 3B; representative (benzimidazolyl)isoquinolinols shown). With the exception of DPCPX, it was not possible to calculate a $\mathrm{p} K_{\mathrm{i}}$ value for all compounds studied (DPCPX $\mathrm{p} K_{\mathrm{i}} ; 8.82 \pm 0.16$ ). To our surprise, no displacement of CA200645 was observed when using 1, which was previously reported as a high affinity and selective $\mathrm{A}_{1} \mathrm{AR}$ ligand ( $\mathrm{hA}_{1} \mathrm{AR} K_{\mathrm{i}}=14.1 \pm 1.6 \mathrm{nM}$, Fig. 1 ) in a $\left[{ }^{3} \mathrm{H}\right] \mathrm{DPCPX}$ binding assay in membranes obtained from CHO cells stably expressing hA1AR. ${ }^{8}$ Interestingly both 1-(1H-1,3-benzodiazol-2yl)isoquinolin-6-ol 17 and 3-(6-methyl-1 $H$-1,3-benzodiazol-2-yl) isoquinolin-6-ol 37 both showed better (although still limited) binding to NLuc $\mathrm{A}_{1} \mathrm{AR}$ than 1 (Fig. 3B).

\section{Experimental}

Full details of the synthesis of 1, 4-43, including all methodology, chemical synthesis, pharmacology, equipment and compound characterisation can be found in the ESI, $\dagger$ along with a comprehensive tautomer study of 18. All compounds tested in the BRET assay were shown by analytical HPLC to possess $>95 \%$ purity. As a representative examples of a DDQ aromatisation reaction, conversion of 6 to 7 was as follows: to a solution of $6(0.17 \mathrm{~g}, 0.83 \mathrm{mmol})$ in 1,4-dioxane : THF $(10 \mathrm{~mL}$ $1: 1, \mathrm{v} / \mathrm{v})$ at $45{ }^{\circ} \mathrm{C}$ was added DDQ $(0.38 \mathrm{~g}, 1.67 \mathrm{mmol})$ and the reaction was stirred vigorously at $45^{\circ} \mathrm{C}$ for $5 \mathrm{~h}$ with the mouth of the flask open to the atmosphere to allow mixing of air. 1,4Dioxane $(10 \mathrm{~mL})$ was added and the reaction mixture filtered, the filtrate was diluted with EtOAc and washed three times with sat aq. $\mathrm{NaHCO}_{3}$ solution. The organic washings were combined, washed once with water, brine solution, then dried over $\mathrm{MgSO}_{4}$, concentrated under reduced pressure and purified by silica gel flash column chromatography $(30-50 \%$ EtOAc/hexane) to provide 7 (84 mg, $0.413 \mathrm{mmol}$, yield $49 \%$ ) as an off-white solid. ${ }^{1} \mathrm{H}$ NMR (400 MHz, MeOD- $\left.d_{4}\right) \delta 4.04\left(\mathrm{~s}, 3 \mathrm{H}, \mathrm{OCH}_{3}\right), 7.16(\mathrm{~d}, J=$ $2.5 \mathrm{~Hz}, 1 \mathrm{H}, \mathrm{ArH}), 7.28(\mathrm{dd}, 1 \mathrm{H}, J=2.5,9.3 \mathrm{~Hz}, \mathrm{ArH}), 7.73(\mathrm{~d}, 1 \mathrm{H}, J$ $=5.7 \mathrm{~Hz}, \operatorname{ArH}), 8.30$ (d, $1 \mathrm{H}, J=5.7 \mathrm{~Hz}, \operatorname{ArH}), 8.52$ (d, $1 \mathrm{H}, J=$ $9.3 \mathrm{~Hz}, \mathrm{ArH}) .{ }^{13} \mathrm{C}$ NMR $\left(101 \mathrm{MHz}, \mathrm{MeOD}-d_{4}\right) \delta 53.24,108.72$, 122.70, 122.89, 124.25, 129.49, 141.07, 141.83, 149.28, 161.18, 167.61. HRMS calculated for $\mathrm{C}_{11} \mathrm{H}_{10} \mathrm{NO}_{3}(\mathrm{M}+\mathrm{H})^{+}, 204.0655$; found, 204.0647.

\section{Conclusions}

Our overall goal was to design new $\mathrm{A}_{1} \mathrm{AR}$ selective fluorescent ligands, however it can be concluded that a (benzimidazolyl)isoquinolinol scaffold linked via the C5 or C6 isoquinoline position to a fluorophore is not suitable for this purpose. Although exploration of other regions of the (benzimidazolyl)isoquinoline scaffold (such as from the benzimidazole) for linker attachment are possible, in light of the poor $\mathrm{A}_{1} \mathrm{AR}$ binding observed for $\mathbf{1}$, the selection of a different $\mathrm{A}_{1} \mathrm{AR}$ selective pharmacophore may be optimal. Although the end biological aim of $\mathrm{A}_{1} \mathrm{AR}$ fluorescent ligands was not achieved, a method for aromatisation of tetrahydroisoquinolinols to give isoquinolinols and conformational (tautomer) studies of (benzimidazolyl)isoquinolinols are reported. 
Since there are only two reports ${ }^{25,43}$ for non-metal-mediated aromatisation of unprotected tetrahydroisoquinolinol to isoquinolinol, for which experimental details are unclear and no yields are provided, the method described herein using DDQ and atmospheric oxygen in mild conditions will prove useful for the preparation of biologically important isoquinolines and isoquinolinols.

\section{Conflicts of interest}

There are no conflicts to declare.

\section{Acknowledgements}

This work was supported by a University of Otago Research Grant and the School of Pharmacy, University of Otago and the University of Nottingham. S. S. is supported by a Doctoral Scholarship from the School of Pharmacy, University of Otago. The authors thank Dr Leigh Stoddart of the Physiology, Pharmacology and Neuroscience group at the University of Nottingham for making the NLuc $\mathrm{A}_{3} \mathrm{AR}$ construct and Promega Corporation for making the NLuc $\mathrm{A}_{1} \mathrm{AR}$ construct.

\section{References}

1 J.-F. Chen, H. K. Eltzschig and B. B. Fredholm, Nat. Rev. Drug Discovery, 2013, 12, 265-286.

2 C. E. Müller and K. A. Jacobson, Biochim. Biophys. Acta, 2011, 1808, 1290-1308.

3 L. A. Stoddart, C. W. White, K. Nguyen, S. J. Hill and K. D. Pfleger, Br. J. Pharmacol., 2016, 173, 3028-3037.

4 E. Kozma, P. S. Jayasekara, L. Squarcialupi, S. Paoletta, S. Moro, S. Federico, G. Spalluto and K. A. Jacobson, Bioorg. Med. Chem. Lett., 2013, 23, 26-36.

5 A. J. Vernall, S. J. Hill and B. Kellam, Br. J. Pharmacol., 2014, 171, 1073-1084.

6 M. Macchia, F. Salvetti, S. Barontini, F. Calvani, M. Gesi, M. Hamdan, A. Lucacchini, A. Pellegrini, P. Soldam and C. Martini, Bioorg. Med. Chem. Lett., 1998, 8, 3223-3228.

7 A. J. Vernall, L. A. Stoddart, S. J. Briddon, H. W. Ng, C. A. Laughton, S. W. Doughty, S. J. Hill and B. Kellam, Org. Biomol. Chem., 2013, 11, 5673-5682.

8 B. Cosimelli, S. Taliani, G. Greco, E. Novellino, A. Sala, E. Severi, F. Da Settimo, C. La Motta, I. Pugliesi, L. Antonioli, M. Fornai, R. Colucci, C. Blandizzi, S. Daniele, M. L. Trincavelli and C. Martini, ChemMedChem, 2011, 6, 1909-1918.

9 Z.-G. Gao, J. E. Van Muijlwijk-Koezen, A. Chen, C. E. Müller, A. P. Ijzerman and K. A. Jacobson, Mol. Pharmacol., 2001, 60, 1057-1063.

10 L. H. Heitman, A. Goblyos, A. M. Zweemer, R. Bakker, T. Mulder-Krieger, J. P. van Veldhoven, H. de Vries, J. Brussee and A. P. IJzerman, J. Med. Chem., 2009, 52, 926931.

11 Z. Ma, L. Du and M. Li, J. Med. Chem., 2014, 57, 8187-8203.
12 L. A. Stoddart, E. K. M. Johnstone, A. J. Wheal, J. Goulding, M. B. Robers, T. Machleidt, K. V. Wood, S. J. Hill and K. D. G. Pfleger, Nat. Methods, 2015, 12, 661-663.

13 E. D. Cox and J. M. Cook, Chem. Rev., 1995, 95, 1797-1842. 14 H.-P. Buchstaller, L. Burgdorf, D. Finsinger, C. Amendt, M. Grell, C. Sirrenberg, F. Zenkeand Preparation of isoquinoline derivatives as kinase inhibitors, WO2005082858A2, Merck Patent Gmbh, 2005.

15 H.-P. Buchstaller, L. Burgdorf, D. Finsinger, F. Stieber, C. Sirrenberg, C. Amendt, M. Grell, F. Zenke and M. Krier, Bioorg. Med. Chem. Lett., 2011, 21, 2264-2269.

16 K. Iwasa, M. Moriyasu, Y. Tachibana, H.-S. Kim, Y. Wataya, W. Wiegrebe, K. F. Bastow, L. M. Cosentino, M. Kozuka and K.-H. Lee, Bioorg. Med. Chem., 2001, 9, 2871-2884.

17 F. Navas and P. K. Spearing, Preparation of isoxazole compounds as therapeutic farnesoid $\mathrm{X}$ receptor agonists, US pat., 20080096921A1, Smithkline Beecham Corporation, 2008.

18 E. F. Calderwood, M. Duffey, A. E. Gould, P. D. Greenspan, B. Kulkarni, M. J. Lamarche, R. S. Rowland, M. Tregay, and T. J. Vos, Bicyclic compounds with kinase inhibitory activity, WO2007067444A1, Millennium Pharmaceuticals, Inc., 2007.

19 S. Chakraborty, W. W. Brennessel and W. D. Jones, J. Am. Chem. Soc., 2014, 136, 8564-8567.

20 W. Yao, Y. Zhang, X. Jia and Z. Huang, Angew. Chem., Int. Ed., 2014, 53, 1390-1394.

21 R. Chen, J. Qi, Z. Mao and S. Cui, Org. Biomol. Chem., 2016, 14, 6201-6204.

22 A. M. M. Mjalli, D. Jones, D. R. Gohimmukkula, G. Huang, J. Zhu, M. Rao, R. C. Andrews, and T. Ren, Benzazole derivatives, compositions, and methods of use as bsecretase inhibitors, WO2006099379A2, Transtech Pharma, Inc., 2006.

23 T. R. Burke Jr, P. L. Russ and V. E. Marquez, Heterocycles, 1992, 34, 757-764.

24 A. Jirgensons, E. Loza, M. Charlton, P. W. Finn, D. P. L. Ribas, and A. Saint-Leger, Novel N-acylarylsulfonamide derivatives as aminoacyl-tRNA synthetase inhibitors, WO2016129983A1, Latvian Institute Of Organic Synthesis, 2016.

25 A. Abeywardane, S. R. Brunette, M. J. Burke, T. M. Kirrane Jr, C. C. Man, D. R. Marshall, A. K. Padyana, H. Razavi, R. Sibley, K. L. L. Smith, R. J. Snow, R. J. Sorcek, H. Takahashi, S. J. Taylor, M. R. Turner, E. R. R. Young, Q. Zhang, Y. Zhang, and R. M. Zindell, Pyrazole derivatives which inhibit leukotriene production, WO2014014874A1, Boehringer Ingelheim International Gmbh, 2014.

26 V. K. Tiwari, N. Kamal and M. Kapur, Org. Lett., 2017, 19, 262-265.

27 A. G. Schultz, W. G. McMahon, R. R. Staib and R. K. Kullnig, Tetrahedron Lett., 1987, 28, 4929-4932.

$28 \mathrm{~J}$. J. Li and J. A. Tino, Tetrahydroisoquinoline analogs useful as growth hormone secretagogues, WO2001085695A1, Bristol-Myers Squibb Co., 2001.

29 M. C. Maillard, F. A. Brookfield, S. M. Courtney, F. M. Eustache, M. J. Gemkow, R. K. Handel, 
L. C. Johnson, P. D. Johnson, M. A. Kerry, F. Krieger, M. Meniconi, I. Muñoz-Sanjuán, J. J. Palfrey, H. Park, S. Schaertl, M. G. Taylor, D. Weddell and C. Dominguez, Biorg. Med. Chem., 2011, 19, 5833-5851.

30 D. Ma, W. Wu, G. Yang, J. Li, J. Li and Q. Ye, Bioorg. Med. Chem. Lett., 2004, 14, 47-50.

31 P. L. Ornstein, M. B. Arnold, N. K. Augenstein and J. W. Paschal, J. Org. Chem., 1991, 56, 4388-4392.

32 A. M. M. Mjalli, D. Jones, D. R. Gohimmukkula, G. Huang, J. Zhu, M. Rao, R. C. Andrews, and T. Ren, Benzazole derivatives, compositions, and methods of use as bsecretase inhibitors, WO2006099379A2, Transtech Pharma, Inc., 2006.

33 S. Seiwert, L. Beigelman, B. Buckman, V. Serebryany, and A. D. Stoycheva, Preparation of proline tripeptides and analogs as inhibitors of hepatitis $\mathrm{C}$ virus replication for treating hepatitis $\mathrm{C}$ infection and liver fibrosis, WO2010045266A1, InterMune, Inc., USA, 2010.

34 D. S. Karanewsky, J. Fotsing, C. Tachdjian, and M. Arellano, Identification of human $\mathrm{T} 2 \mathrm{R}$ receptors that respond to bitter compounds that elicit the bitter taste in compositions, and the use thereof in assays to identify compounds that inhibit (block) bitter taste in compositions and use thereof, WO2011106114A1, Senomyx, Inc., USA, 2011.

35 S. Ishiguro, N. Kawaguchi, M. Nakakoshi, S. Shimada, M. Seya, M. Nomoto, M. Okue, and H. Tomizuka, Preparation of isoquinoline derivatives as protease inhibitors, JP07304744A, Snow Brand Milk Prod Co Ltd, Japan, 1995.

36 S. Chen, Y. Li, P. Ni, B. Yang, H. Huang and G.-J. Deng, J. Org. Chem., 2017, 82, 2935-2942.

37 T. Shamim, M. Gupta and S. Paul, J. Mol. Catal. A: Chem., 2009, 302, 15-19.

38 J. Dong, X.-X. Shi, J.-J. Yan, J. Xing, Q. Zhang and S. Xiao, Eur. J. Org. Chem., 2010, (36), 6987-6992.

39 A. S.-K. Tsang, P. Jensen, J. M. Hook, A. S. K. Hashmi and M. H. Todd, Pure Appl. Chem., 2011, 83, 655-665.

40 S. Höck, R. Marti, R. Riedl and M. Simeunovic, CHIMIA International Journal for Chemistry, 2010, 64, 200-202.

41 L. A. Stoddart, L. E. Kilpatrick and S. J. Hill, Trends Pharmacol. Sci., 2018, 39, 136-147.

42 M. A. Arruda, L. A. Stoddart, K. Gherbi, S. J. Briddon, B. Kellam and S. J. Hill, Front. Pharmacol., 2017, 8, 908.

43 B. Franck, G. Blaschke and G. Schlingloff, Angew. Chem., 1963, 75, 957-965. 